\title{
MRI 低噪声前置放大器设计研究
}

\author{
曹学明，组栋林"，赵旭娜，范阳，高家红 \\ 北京大学物理学院重离子所医学物理和工程北京市重点实验室, 北京 100871 \\ *E-mail:dlzu@pku.edu.cn
}

收稿日期: 2010-11-14; 接受日期: 2011-02-21

国家自然科学基金资助项目(批准号: 60871001)

\begin{abstract}
摘要本文研究、设计了一个可用于 MRI 的低噪声前置放大器. 提出了一个 3 元件的噪声 匹配网络方案, 用以变换 $50 \Omega$ 信号源阻抗为 FET 的最佳源阻抗, 以使前置放大器的噪声系数 达到最小, 并具有可观的增益. 实验证明前置放大器输入端在阻抗匹配条件下可获得最大增 益但噪声系数不小. 用砷化镓场效应管(AsGa-FET)ATF33143 作为放大管制作的单级低噪声 前置放大器在 $128 \mathrm{MHz}(3 \mathrm{~T})$ 频率上用网络分析仪(HP8712C)测量增益可以达到 $25 \mathrm{~dB}$, 用噪 声系数分析仪(8970B)测量的噪声系数为 $0.43 \mathrm{~dB}$.
\end{abstract}

关键词

MRI

噪声系数 前置放大器 噪声匹配网络 源阻抗
众所周知, 核磁共振成像(MRI)信号是微伏量级 的信号, 即接收线圈得到的信号相当微弱, 必须经过 几个量级的放大后才能进行正交相敏检波 ${ }^{[1]}$ 、正交数 字化采集 ${ }^{[2]}$ 等处理. 对于 $\mathrm{MRI}$ 来说, 信噪比 $S N R$ 是一 个永恒的研究课题. 根据 NMR 信噪比公式 ${ }^{[3,4]}$, 有多 个提高 $S N R$ 的途径, 比如把主磁场 $B_{0}$ 翻番, $S N R$ 可以 提高到 2 2.8 倍, 取决于生理噪声是否居支配地位; 使用正交解调比非正交解调 $S N R$ 可提高到 1.4 倍. 上 述 $S N R$ 都是指本征信噪比, 即由接收线圈得到的信 噪比, 任何一点提高都要付出很大的代价, 因此本征 信噪比是极其宝贵的. NMR 信号由接收线圈获得, 在接下来的放大过程中, 信噪比总是会不同程度地 降低. 也就是说, 每一级放大器都要增加一些额外的 噪声, 使得最后的图像信噪比总是低于本征信噪比. 为了减缓 $S N R$ 变坏的程度, 前人研究结果表明: 每一 级放大器带入信号中的噪声并不是平均分配的. 定 义放大器噪声系数为 $N_{\mathrm{F}}=\frac{(S / N)_{\text {in }}}{(S / N)_{\text {out }}} . N_{\mathrm{F}}$ 是一个大于 1
的数. 多级放大器级联时, 总噪声系数 $N_{F}$, 即首级输 入信噪比, 与末级输出信噪比有如下关系 ${ }^{[4]}$ :

$$
N_{\mathrm{F}}^{2}=N_{\mathrm{f} 1}^{2}+\frac{N_{\mathrm{f} 2}^{2}}{G_{1}^{2}}+\frac{N_{\mathrm{f} 3}^{2}}{G_{1}^{2} \cdot G_{2}^{2}}+\ldots,
$$

式中 $N_{\mathrm{f} 1}, G_{1}$ 分别是第 1 级放大器的噪声系数和增益, 依次类推. 可见头一级放大器的噪声系数占据了绝 对的支配地位. 只要把第 1 级放大器的噪声系数做得 足够低, 增益足够高, 不但其后面级联放大器贡献的 噪声完全不必考虑, 还能保证最终可用信号的信噪 比不比本征 $S N R$ 降低很多. 这个第 1 级放大器通常被 称谓“低噪声前置放大器”. 如果这一级噪声系数为 $0.5 \mathrm{~dB}$, 增益 $25 \mathrm{~dB}$, 则通过这一级放大器后信噪比 仅下降 $5.6 \%$, 第二级放大器噪声系数的贡献也可以 忽略. 如果这一级 $N_{\mathrm{F}}=6 \mathrm{~dB}$, 则信噪比损失将达到 $50 \%$. 这就意味着将主磁场 $B_{0}$ 从 $1.5 \mathrm{~T}$ 升到 $3.0 \mathrm{~T}$ 的 巨大努力等于白费.

MRI 低噪声前置放大器的设计是一项专门技术, 对它提出了不同于其他放大器的要求. 就空间位置 
来说, 它紧邻射频(RF) 接收线圈或发射/接收两用线 圈. 如果把线圈接收到的 NMR 信号直接经过长电缆 从磁体室引出到射频室的放大器, 会引起相当大的 衰减, 产生信噪比的严重损失. 因此通常是把线圈接 收到的微弱信号先通过低噪声前置放大器放大一定 倍数后, 再经长电缆线传输到后续级联的放大器进 一步放大. 一般说, 低噪声前置放大器在保证一定放 大倍数的前提下一定要有很低的噪声系数 $(\leqslant 0.5 \mathrm{~dB})$. 除此以外, 根据特定的条件对低噪声前置放大器还 有某些特定的要求. 例如对于相位阵列接收线圈、并 行采集系统, 常把低噪声前置放大器置于各线圈内, 没有 $50 \Omega$ 的电缆传输, 不需要考虑 $50 \Omega$ 的阻抗匹配, 可直接考虑噪声匹配. 有时, 考虑到阵列线圈中间隔 线圈之间的退耦(也叫去耦), Roemer 等人 ${ }^{[5]}$ 把低噪声前 置放大器的输入阻抗做得很低 (5 $\Omega$ 以内). Reykowski 等人 ${ }^{[6]}$ 设计了 4 元件噪声匹配网络故意失配源阻抗和 前置放大器输入阻抗以进行相位阵列中线圈之间的 退耦. 另外, 也有用电容退耦机制和 $50 \Omega$ 接收机建 立并行采集最佳阵列方案 ${ }^{[7]}$.

MRI 早期, 曾考虑为了使线圈接收到的信号在 低噪声前置放大器处的反射损失最小, 以得到较大 的放大倍数, 前置放大器的输入电路采用阻抗匹配 的设计方法 ${ }^{[3]}$, 这样就势必增大了噪声系数. 有些特 殊场合可以通过降低前置放大器的温度来降低前置 放大器的噪声系数 ${ }^{[8]} . \mathrm{S}$ 参数和噪声参数的计算测量 对设计高质量前置放大器是必不可少的 ${ }^{[9]}$, 然而场效 应晶体管的 datasheet 中给出的 $\mathrm{S}$ 参数数据几乎全部 都是在很高频率段下测量的, 根本不覆盖磁共振系 统所需要的工作频段. 这给设计 MRI 前放造成极大 的不便.

本文设计的 MRI 低噪声前置放大器, 由于将用 于 $3 \mathrm{~T}$ 系统上一个发射/接收( $\mathrm{T} / \mathrm{R})$ 两用线圈, 线圈需 要先匹配到 $50 \Omega$ 电缆以便接收 RF 功率, 射频通路经 过 RF 开关转换发射/接收两种状态. 因此, 这就限定 了前置放大器的信号源阻抗是 $50 \Omega$. 考虑只用一级 FET 进行放大, 噪声系数尽可能低, 但需兼顾一定的 增益, 以使第二级放大器的噪声可完全忽略.

\section{1 低噪声前置放大器电路设计}

MRI 低噪声前置放大器是一个小信号模拟放大 器, 其电路原理方框图如图 1 所示. 主要由输入级噪

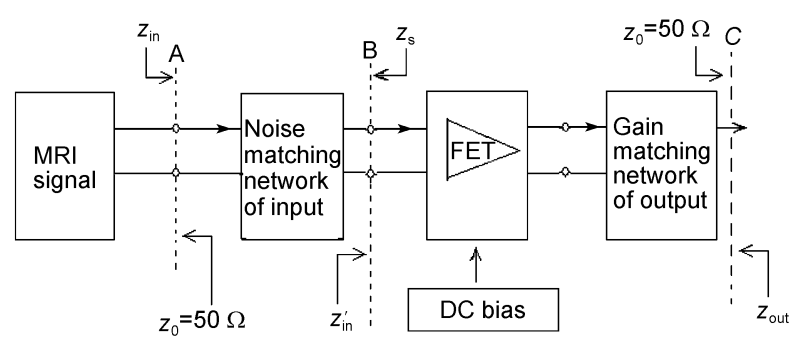

图 1 低噪声前置放大器的结构框图

声匹配网络、放大管和输出级增益匹配网络组成. 对 于放大器, 根据低噪声要求和市场调查, 砷化镓场效 应管(AsGa-FET) 是噪声最低的有源器件, 当为首选. 而砷化镓场效应管产品型号很多, 基本都是用于微 波波段(1 100 GHz)的器件, MRI 工作波段(10 400 $\mathrm{MHz}$ )虽然也是最佳可选器件, 但是很遗憾, 几乎完 全没有这个频段的数据. 本文试验了两种型号, 工作 在 $128 \mathrm{MHz}$ 时型号 ATF33143 结果更优越一些, 它是 低噪声前置放大器的核心元件. 根据该产品数据手 册(datasheet $)^{[10]}$, 考虑使噪声系数尽可能低, 但又需 兼顾一定增益和热稳定性, 本文选择的直流偏置条 件为 $V_{\mathrm{GS}}=-0.48 \mathrm{~V}, V_{\mathrm{DS}}=2.13 \mathrm{~V}, I_{\mathrm{DS}}=44 \mathrm{~mA}$. 根据 ATF33143 的 Datasheet 给出的各频段噪声参数 ${ }^{[10]}$, 外 推求得在 $128 \mathrm{MHz}$ 下的噪声参数, 作为设计噪声匹 配网络的依据.

\subsection{FET 的偏置和匹配网络}

场效应晶体管 ATF33143 的输入级匹配网络可 以是增益匹配网络, 也可以是噪声匹配网络, 但不可 能同时兼是二者. 当然可以同时兼顾二者, 但那叫折 衷. 因此可以把放大管输入级阻抗变换为 $Z_{\text {in }}=50 \Omega$, 与线圈要求的 $50 \Omega$ 电缆传输线相匹配(图 1), 以获取 最大增益. 也可以把信号源阻抗 $50 \Omega$ 变换为放大管 的最佳源阻抗 $Z_{\mathrm{s}}$ (图 1), 以获取最佳噪声特性. 一般 来说, MRI 前放的输入级要求是后者, 因此叫噪声匹 配网络. 这类匹配网络并不唯一, 可以用 3 个分立元 件构成也可以用 4 个分立元件 ${ }^{[6]}$ 构成, 然而 2 个元件 似乎太少, 难以达到目的. 本文采用了 2 个电容和 1 个电感共 3 个元件构成输入匹配网络, 如图 2 所示. 这样电感 $L_{1}$ 兼起直流接地、电容 $C_{1}$ 兼起隔直流作用, 它们与源极电阻 $R_{\mathrm{e}}$ 一起实现对放大管的直流偏置, $r$ 为电感 $L_{1}$ 的趋肤电阻, $C_{3}$ 起高频旁路作用. 放大管输 出网络为增益匹配网络, 由 $L_{3}, C_{4}, L_{2}$ 和 $C_{5}$ 组成, 把 


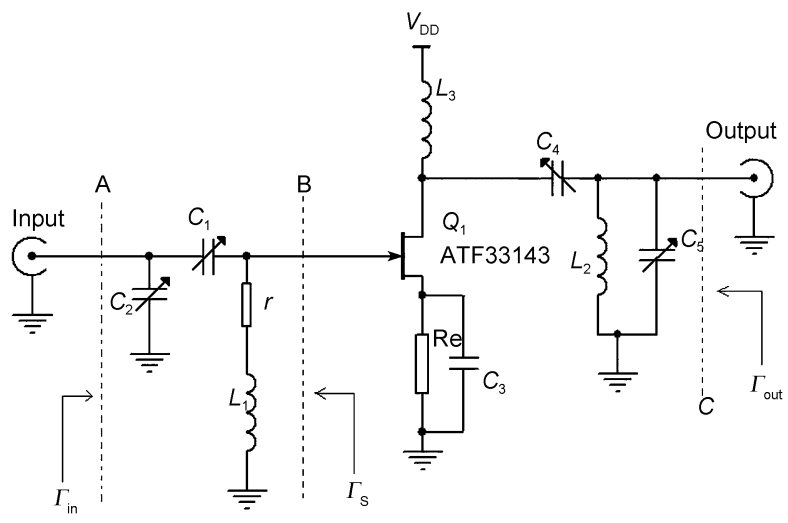

图 2 低噪声前置放大器偏置电路和匹配网络

放大管输出阻抗匹配到 $50 \Omega$, 由 $50 \Omega$ 长电缆引出磁 体室到后续放大器.

\section{2 噪声和增益考虑}

一般说 FET 管的输入阻抗 $Z_{\text {in }}^{\prime}$ 很高, 在 $10 \mathrm{M} \Omega$ 量 级, 直接匹配其输入阻抗是困难的. 然而通过上述一 个三元件输入网络如图 2 所示, 令该网络输入阻抗等 于 $50 \Omega$, 与线圈信号 $50 \Omega$ 输出电缆相匹配是不难做 到的. 直流偏置保持为 $V_{\mathrm{DS}}=2.13 \mathrm{~V}, I_{\mathrm{DS}}=44 \mathrm{~mA}$ 不变. 用网络分析仪(HP8712C)进行测量, 在 $128 \mathrm{MHz}$ 时调 节可变电容 $C_{1}$ 和 $C_{2}$, 观察低噪声前置放大器增益随 之变化的情况. 当增益取到最大值 $37 \mathrm{~dB}$ 时, 通过 Smith 圆图可以看到此时输入网络的输入阻抗 $Z_{\text {in }}$ 匹 配到了 $50 \Omega$. 此时测量输入网络反射系数(图 2), 结 果是 $\Gamma_{\mathrm{in}}=0$, 满足关系式 $G_{\mathrm{in}}=\left(Z_{\mathrm{in}}-50\right) /\left(Z_{\mathrm{in}}+50\right)$. 然 而噪声系数却为 $1.74 \mathrm{~dB}$, 远离理想值. 理论上讲, 增 益最大和噪声最小也是不可能同时达到的. 理论上 前置放大器的增益可以表示为 ${ }^{[11]}$

$$
A=A_{0}\left(1-\left|\Gamma_{\text {in }}\right|^{2}\right),
$$

放大器噪声系数 $N_{\mathrm{F}}$ 由下式 ${ }^{[10,11]}$ 确定:

$$
N_{\mathrm{F}}=\left(N_{\mathrm{F}}\right)_{\min }+\frac{4 R_{\mathrm{n}}}{Z_{0}} \frac{\left|\Gamma_{\mathrm{s}}-\Gamma_{\mathrm{opt}}\right|^{2}}{\left(\left|1+\Gamma_{\mathrm{opt}}\right|^{2}\right)\left(1-\left|\Gamma_{\mathrm{s}}\right|^{2}\right)},
$$

式中 $R_{\mathrm{n}}$ 为放大管等效噪声电阻, $Z_{0}$ 为电缆阻抗 $50 \Omega$, $\Gamma_{\mathrm{s}}$ 为放大管的源反射系数如图 2 所示. $\Gamma_{\mathrm{opt}}$ 是放大管 的最佳源反射系数. 当源反射系数 $\Gamma_{\mathrm{s}}$ 等于放大管所 要求的最佳源反射系数 $\Gamma_{\mathrm{opt}}$ 时, 放大器具有最小噪声 系数 $\left(N_{\mathrm{F}}\right)_{\min }$. 输入网络的作用就是把线圈信号源阻抗 $50 \Omega$ 变换为放大管要求的最佳源阻抗 $Z_{\mathrm{s}}($ 图 1). 厂商
在 ATF33143 管的数据手册中给出了 $500 \mathrm{MHz} 10$ $\mathrm{GHz}$ 频率范围内 $N_{\mathrm{F} \mathrm{min}}, R_{\mathrm{n}} / 50$ 和 $\Gamma_{\mathrm{opt}}$ 的测量值, 本文外 推到 $128 \mathrm{MHz}$ 得到一套数据, 作为参考. 以求配置到 最佳 $\Gamma_{\mathrm{s}}$ (图 2). 最佳源反射系数 $\Gamma_{\mathrm{opt}}$ 与这个噪声匹配 网络的 $Z_{\mathrm{s}}$ 的最佳值 $Z_{\mathrm{opt}}$ 有关, 而 $Z_{\mathrm{s}}$ 由电容 $C_{1}, C_{2}$ 和电 感 $L_{1}$ 确定

$$
Z_{\mathrm{s}}=\left(\left(50 \| Z_{C_{2}}\right)+Z_{C_{1}}\right) \|\left(r+Z_{L_{1}}\right),
$$

式中 $Z_{\mathrm{L}_{1}}=j \omega L_{1}, Z_{\mathrm{C}_{1}}=\frac{1}{j \omega C_{1}}, Z_{\mathrm{C}_{2}}=\frac{1}{j \omega C_{2}}, \|$ 号表示并联.

倘若知道 $C_{1}, C_{2}$ 和 $L_{1}$ 的值, 可以计算出 $Z_{\mathrm{s}}$, 然而 手册中并未给出最佳值 $Z_{\mathrm{opt}}$. 放大管的输入阻抗 $Z_{\mathrm{in}}^{\prime}$ 在 datasheet 中也从来不给出. 因此无法运用 $\Gamma_{\mathrm{s}}=$ $\left(Z_{\mathrm{s}}-Z_{\text {in }}^{\prime}\right) /\left(Z_{\mathrm{s}}+Z_{\text {in }}^{\prime}\right)$ 计算 $\Gamma_{\mathrm{s}}$. 手册中虽然给出了 $\Gamma_{\mathrm{s}}$ 的 最佳值 $\Gamma_{\mathrm{opt}}$, 然而直接测量 $\Gamma_{\mathrm{s}}$ 并不方便. 但借助于噪 声系数仪, 可通过调整电容 $C_{1}, C_{2}$ 和 $L_{1}$ 使放大器噪声

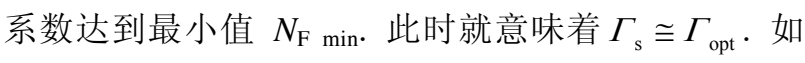
果此时前置放大器增益满足要求, 那是最希望的. 如 果增益不满足要求, 就需要进行折衷, 即适当牺牲噪 声性能而提高增益, 以使两者都满足要求.

\section{2 电路调试及实验测量结果}

设计完成的前置放大器电路如图 3 所示. 其中电 感 $L_{3}$ 、晶体管 $Q_{2}$ 、稳压电源 7805 及周围电阻、电容 构成 FET 放大管的直流供电电路. $L_{3}$ 对高频起扼流作 用, $R_{1}$ 是 $Q_{2}$ 的偏置电阻, $Q_{2}$ 基极对地电容 $C_{6}$ 经晶体 管放大后容量增大许多倍, 可以有效地滤除较高频 率的纹波. 电容 $\mathrm{C}_{7} \sim \mathrm{C}_{10}$ 都是滤波电容, 考虑到不同容 量电容对频率的敏感性, 故用不同容量的电容并联. 二极管在外接电源极性反向的情况下可起保护作用. 电路其他部分都与图 2 相同, 通过多次调试确定了最 终元件值. 电路焊接完成后装在一个小屏蔽盒内, 进 行了多次调试. 当输入网路取 $C_{1}=5.5 \mathrm{pF}, C_{2}=60 \mathrm{pF}$ 和 $L_{1}=0.12 \mu \mathrm{H}$ 时, 输出网络取 $C_{4}=70 \mathrm{pF}, C_{5}=30 \mathrm{pF}$ 和 $L_{1}=0.1 \mu \mathrm{H}$ 时的增益最大, 前已提及为 $A_{0}=37 \mathrm{~dB}$. 然 而此时的噪声系数却为 $1.74 \mathrm{~dB}$, 显然不符合低噪声 前置放大器的要求, 而且稳定性也难以保证.

保持输出网络不变, 把 $L_{1}$ 调整为 $L_{1}=0.15 \mu \mathrm{H}$, 再 调整可变电容 $C_{1}$ 和 $C_{2}$, 观察噪声系数的变化. 结果 发现当 $C_{1}=15 \mathrm{pF}, C_{2}=10 \mathrm{pF}$ 时, 前置放大器噪声系数 降低到 $0.43 \mathrm{~dB}$, 增益为 $25 \mathrm{~dB}$. 图 4 (照片)显示的是 


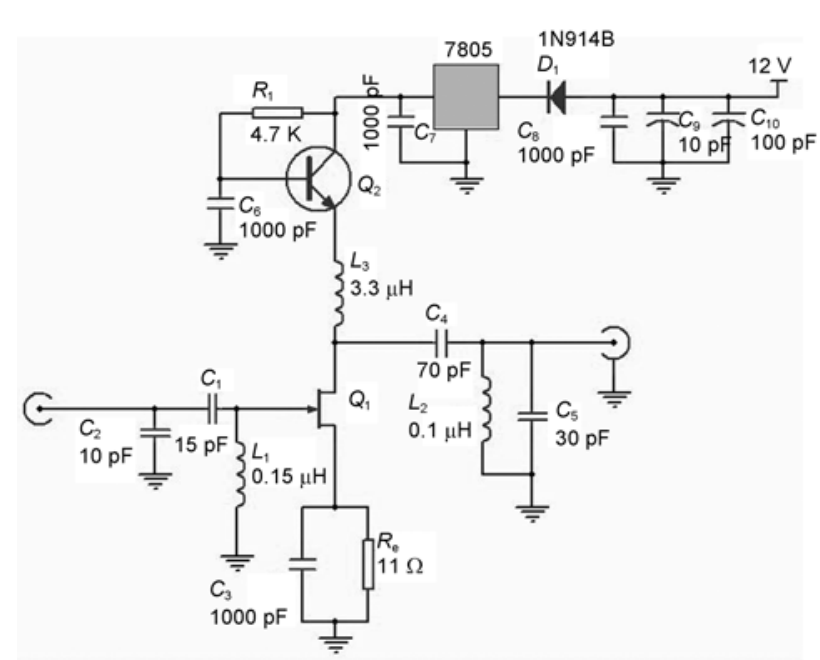

图 3 低噪声前置放大器的电路原理图

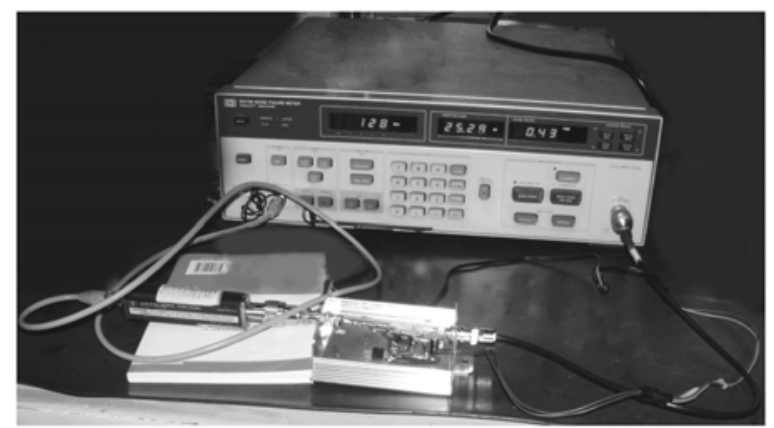

图 4 在屏蔽间内用 HP8970B 噪声系数仪测量自制前放 的增益和噪声系数的照片

用噪声系数仪(HP8970B)测量前置放大器在 $128 \mathrm{MHz}$ 时增益和噪声系数的结果. 实际上, 对前置放大器在 各种频率下的增益和噪声系数进行了系统的测量, 结果显示在图 5 中. 图中显示噪声系数和增益随频率 增大而几乎线性下降. 这是由场效应管的特性决定 的. 这种管子更适合高频, 就是指随频率增高其噪声 系数越来越低. 前面已经提及, 这类砷化镓 FET 管更 适合用于微波波段, MRI工作频段虽然可用, 但在生 产厂家提供的器件手册中没有这个波段的测量数据.

最后用网络分析仪测量前置放大器在 $128 \mathrm{MHz}$ 时的输出反射系数 $\left|\Gamma_{\mathrm{out}}\right|=0.15$, 输入反射系数 $\left|\Gamma_{\mathrm{in}}\right|=0.97$. 接近于 1 的 $\left|\Gamma_{\mathrm{in}}\right|$ 说明 MRI 低噪声前置放大 器输入匹配网络本质上是噪声匹配网络而不是阻抗 匹配网络. $\left|\Gamma_{\text {out }}\right| \neq 0$ 说明输出网络并没有达到完全匹 配. 如果把 $C_{4}, C_{5}$ 重新换成可调电容, 就可以把 $\Gamma_{\text {out }}$ 调 成 $\mid \Gamma_{\text {out }}=0$, 以达到阻抗完全匹配, 增益还会有所提高.

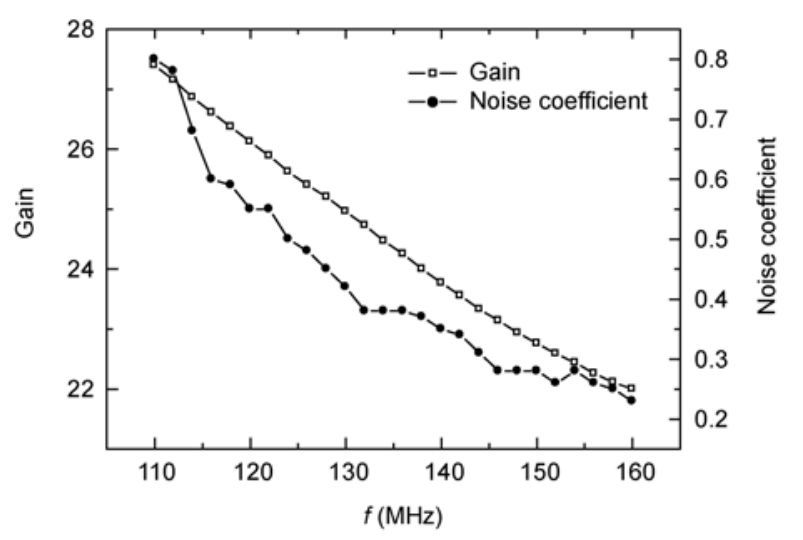

图 5 自制前放的增益和噪声系数随工作频率的变化趋势 圆点代表噪声系数; 方块代表增益

\section{3 讨论}

MRI 本征信噪比取决于主磁场 $B_{0}$ 、样品和 RF 接收线圈 ${ }^{[4]}$. 经低噪声前置放大器后信噪比变差的程 度取决于这一级的噪声系数 $N_{\mathrm{F}}$. 对 $N_{\mathrm{F}}$ 的贡献来自晶 体放大管和输入网络、输出网络以及偏置电路中的电 阻, 其中起支配作用的是晶体管中离子的随机热运 动, 因此输入网络把信号源阻抗匹配到最佳源阻抗 就可以把晶体管对噪声的贡献降到最低. 其次是考 虑匹配网络和偏置电路中的电阻元器件传导电子热 运动对噪声的贡献.

根据(1)式, 输出网络对噪声的贡献可以忽略不 计, 因此输出网络的任务是把晶体管输出阻抗匹配 到 $50 \Omega$ 输出电缆以减小信号反射损失. 为了减小输 入网络的信号损失, 网络元件 $L_{1}, C_{1}, C_{2}$ 应该尽可能 是高 $Q$ 的. 为了偏置晶体管, 不得不使用电阻元件. 然而, 如果电阻元件放在栅极回路, 电阻噪声就会被 放大. 因此偏置电阻置于栅极回路还是源极回路, 其 对噪声的贡献就完全不同. 如果低噪声前置放大器 置于接收线圈内一起放入 MRI 磁孔, 所有元件还应 该是无磁的. 否则, 将影响 MRI 系统 $B_{0}$ 场的均匀性.

影响 MRI 低噪声前置放大器稳定性的因素有晶体 管自激振荡、元件本身温度稳定性差(需要老化)、焊接 质量差、供电电源稳定性差及纹波水平高、接地不可 靠以及屏蔽不善漏进外来 $\mathrm{RF}$ 干扰等, 因此需要在这 诸多方面下足功夫.

当 FET 的 Datasheet 给出的数据不包括前置放大 器工作频率范围时, 如果有条件最好通过测量补充 其工作频率处的数据, 条件不具备时只能用外推的 
数据. 这些外推得到的数据只能作为估算时的参考. 电路应以最后测量调试到最佳状态为准. 输入、输出 网络虽不唯一, 但应以在保证电路功能的前提下包 含尽可能少的元件.

\section{4 结论}

本文成功设计、研制了一个适于 3 T MRI 的单级
低噪声前置放大器, 在 $128 \mathrm{MHz}$ 频率上噪声系数和 增益测量值分别达到 0.43 和 $25 \mathrm{~dB}$. 证明砷化镓场效 应管ATF33143 可以工作在微波低端外延的 $128 \mathrm{MHz}$ 频率上. 如果允许噪声指标可以放宽, 工作在更低频 率上也是可能的(图 5). 运用 3 元件输入网络作为噪 声匹配网络把信号源阻抗 $50 \Omega$ 匹配到 FET 晶体管的 最佳源阻抗是可行的, 而且较为成功, 并仍有进一步 提高性能的余地. 助和支持.

\section{参考文献}

1 姜忠德, 组栋林, 谷晓芳. MRI 系统中正交技术. 北京大学学报(自然科学版), 2006, 42(3): 320-323

2 姜忠德, 组栋林, 谷晓芳. 用于 MRI 系统的正交数据采集卡设计研究. 自然科学进展, 2005, 15(12): 1512-1517

3 Hoult D I, Richards R E. The signal-to-noise ratio of the nuclear magnetic resonance experiment. J Magn Reson, 1976, 24: 71-85

4 组栋林. 核磁共振成像学. 北京: 高教出版社, 2004

5 Roemer P B, Edelstein W A, Hayes C E, et al. The NMR phased array. Magn Reson Med, 1990, 16: 192-225

6 Reykowski A, Wright S M, Porter J R. Design of matching networks for low noise preamplifiers. Magn Reson Med, 1995, 33: 848-852

7 Pinkerton R G, Barberi E A, Menon R S. Noise properties of an NMR transceiver coil array. J Magn Reson, 2004, 171: 151-156

8 Seton H C, Hutchison J M S, Bussell D M. A 4.2 K receiver coil and SQUID amplifier used to improve the SNR of low-field magnetic resonance images of the human arm. Physiol Meas, 2008, 29: R1-R26

9 Doty F D, Entzminger G, Kulkarni J, et al. Radio frequency coil technology for small-animal. NMR Biomedicine, 2007, 20: 304-325

10 Agilent. Low Noise Pseudomorphic HEMT in a Surface Mount Plastic Package (www.agilent.com/semiconductors)

11 Ludwig R, Bretchko P. 王子宇, 张肇仪, 徐承和, 等译. 射频电路设计-理论和应用, 电子工业出版社, 2007 